
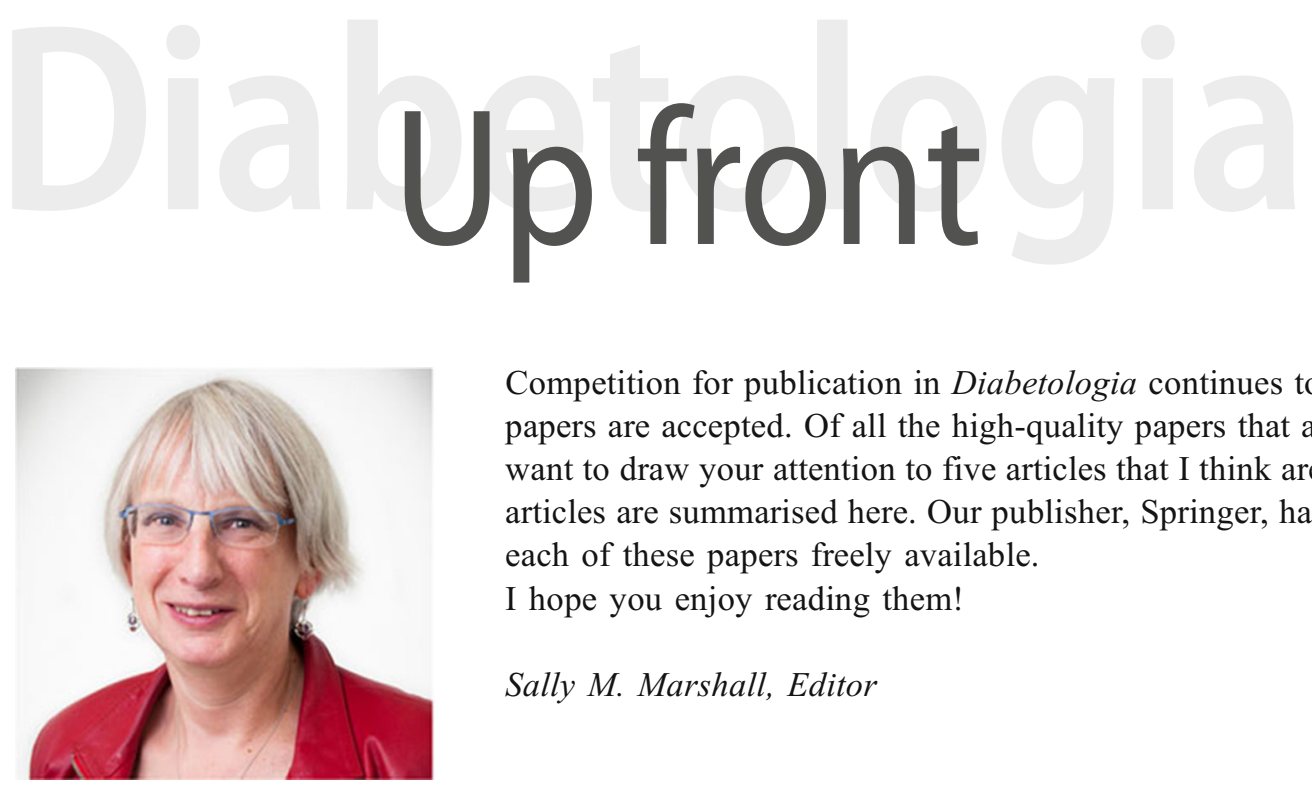

Competition for publication in Diabetologia continues to grow, and less than $20 \%$ of papers are accepted. Of all the high-quality papers that appear in this month's issue I want to draw your attention to five articles that I think are particularly interesting. The articles are summarised here. Our publisher, Springer, has kindly made the full text of each of these papers freely available.

I hope you enjoy reading them!

Sally M. Marshall, Editor

\section{Neoepitopes: a new take on beta cell autoimmunity in type 1 diabetes}

$\overline{\text { Stuart I. Mannering, Anthony R. Di Carluccio, Colleen M. }}$ Elso

T cell responses against neoepitopes are emerging as important players in the development of autoimmune diseases. A variety of mechanisms result in post-translational modification of cellular proteins, leading to the generation of neoepitopes. Some of these 'new' epitopes are specific to peripheral tissues and provide a potential explanation for how self-protein-specific $\mathrm{T}$ cells can avoid thymic deletion. In this issue, Mannering et al (https:// doi.org/10.1007/s00125-018-4760-6) outline recent advances in this field and challenges still to be overcome in the discovery of neoepitopes important in type 1 diabetes. Information about $\mathrm{T}$ cell responses to diabetogenic neoepitopes will support the development of antigen-specific therapies aimed at restoring immune tolerance in type 1 diabetes, as well as assays to monitor function and frequency of antigen-specific cells in the periphery. This may also contribute to the basic understanding of the initiation of autoimmune disease.

\section{Cardiovascular outcome trials of glucose-lowering medi- cations: an update}

\section{Philip Home}

Cardiovascular outcome trials (CVOTs) of glucoselowering medications that have been completed in recent years have provided welcome pointers towards best use of these drugs in diabetes care. However, many questions and uncertainties remained. In this issue, Philip Home (https://doi.org/10.1007/s00125-018-48011) discusses studies in three major glucose-lowering drug classes, which were published and presented recently (October/November 2018). These studies help to confirm or clarify our understanding of how the three classes should be positioned clinically and whether there are within-class differences. For dipeptidyl peptidase-4 (DPP4) inhibitors, CARMELINA confirmed neutrality for cardiovascular outcomes, with no heart failure signal, albeit in a highly selected population. For GLP-1 receptor agonists, the Harmony Outcome study confirmed an early and continuing benefit for cardiovascular protection for this class, independent of glucose- or bodyweight-lowering. For sodium-glucose cotransporter-2 (SGLT2) inhibitors, DECLARE-TIMI 58 confirmed strong renal protection and protection against heart failure, even in people without prior cardiovascular disease, but did not further clarify class issues around major cardiovascular outcomes. Studies in these three classes are now transforming guidelines for the use of glucoselowering medications, notably extending them beyond glucose-lowering to managing the adverse vascular and renal manifestations of diabetes. The three studies discussed here drive guideline changes more firmly for the benefit of people with type 2 diabetes, and related studies due in the next 12 months will take that process further. 
Trends and cyclical variation in the incidence of childhood type 1 diabetes in 26 European centres in the 25 year period 1989-2013: a multicentre prospective registration study

Christopher C. Patterson, Valma Harjutsalo, Joachim Rosenbauer, Andreas Neu, Ondrej Cinek, Torild Skrivarhaug, Birgit Rami-Merhar, Gyula Soltesz, Jannet Svensson, Roger C. Parslow, Conxa Castell, Eugen J. Schoenle, Polly J. Bingley, Gisela Dahlquist, Przemysława K. Jarosz-Chobot, Dalè Marčiulionyte, Edna F. Roche, Ulrike Rothe, Natasa Bratina, Constantin IonescuTirgoviste, Ilse Weets, Mirjana Kocova, Valentino Cherubini, Natasa Rojnic Putarek, Carine E. deBeaufort, Mira Samardzic, Anders Green

Recent publications from Scandinavian countries have suggested that a previously increasing incidence of type 1 diabetes in children under 15 years old may now be stabilising. In this issue, Patterson et al (https://doi.org/10.1007/s00125-0184763-3) report results from a pooled analysis of data from 26 registers in 22 European countries during the 25 year period 1989-2013. The average rate of increase across Europe during this period was estimated as $3.4 \%$ per annum, although different patterns were apparent in different countries. When estimates were obtained for 5 year subperiods, the rate of increase was lowest at $1.1 \%$ per annum in the 2004-2008 period but rose again to $2.7 \%$ per annum in the 2009-2013 period. Evidence of a 4 year periodicity in incidence was evident in four centres but there was no obvious explanation for this. These findings have implications for those involved in the planning and delivery of healthcare for children with type 1 diabetes.

Maternal metabolites during pregnancy are associated with newborn outcomes and hyperinsulinaemia across ancestries

Rachel Kadakia, Michael Nodzenski, Octavious Talbot, Alan Kuang, James R. Bain, Michael J. Muehlbauer, Robert D. Stevens, Olga R. Ilkayeva, Sara K. O’Neal, Lynn P. Lowe, Boyd E. Metzger, Christopher B. Newgard, Denise M. Scholtens, William L. Lowe Jr for the HAPO Study Cooperative Research Group

The maternal metabolome during pregnancy offers insight into the metabolic environment surrounding a developing fetus and may impact on newborn fat deposition and insulin sensitivity. In this issue, Kadakia et al (https://doi.org/10.1007/s00125018-4781-1) investigated these associations in 1600 mothers and offspring who participated in the Hyperglycemia and Adverse Pregnancy Outcome (HAPO) study. Metabolomics assays were performed on maternal fasting and $1 \mathrm{~h}$ post glucose load serum samples. The authors identified several individual maternal fatty acid, lipid and amino acid metabolites at $1 \mathrm{~h}$ post glucose load that were associated with newborn sum of skinfolds, birthweight and cord C-peptide, a measure of fetal insulinaemia. These findings suggest that the maternal metabolomic response to a glucose load may have a greater impact on newborn size than the fasting state. In addition, maternal metabolites may mediate the well-known associations of maternal BMI and maternal glucose with newborn size. A unique maternal metabolomic signature may emerge as an early-life biomarker of offspring obesity risk.

Reduced skeletal-muscle perfusion and impaired ATP release during hypoxia and exercise in individuals with type 2 diabetes

Martin B. Groen, Trine A. Knudsen, Stine H. Finsen, Bente K. Pedersen, Ylva Hellsten, Stefan P. Mortensen

Type 2 diabetes is associated with vascular complications, including reduced blood flow in tissues. Plasma ATP is a potent vasodilator that is thought to play a role in the local regulation of blood flow, and it is possible that ATP signalling is impaired in individuals with type 2 diabetes. In this issue, Groen et al (https://doi.org/10.1007/s00125-018-4790-0) measured femoral arterial and venous plasma ATP levels during normoxia, hypoxia and one-legged knee-extensor exercise in nine individuals with type 2 diabetes and eight control individuals. They report that individuals with type 2 diabetes had a blunted increase in ATP and blood flow, compared with non-diabetic individuals, when exposed to hypoxia (a potent stimulus for ATP release). The authors also found that, compared with the non-diabetic group, individuals with type 2 diabetes had lower venous plasma ATP levels and blood flow in the exercising leg during the one-legged knee-extensor exercise. In additional experiments, the individuals with type 2 diabetes had a lower vasodilatory response to exogenous ATP. Restoration of ATP release (e.g. by phosphodiesterase 3- or phosphodiesterase 5-inhibitors) could, therefore, be a novel treatment to help restore tissue perfusion in individuals with type 2 diabetes.

All text supplied by the authors. 